\title{
Simple complete denture techniques can provide patient satisfaction
}

\author{
When complete dentures are required, are simplified techniques as effective as \\ complex traditional ones for their manufacture?
}

\begin{abstract}
Kawai Y, Murakami H, Shariati B, et al. Do traditional techniques produce better conventional complete dentures than simplified techniques? J Dent 2005; 33:659-668.
\end{abstract}

Design A randomised controlled trial (RCT) was carried out in a hospital environment.

Intervention A total of 122 edentulous individuals, aged $45-75$ years, were randomly allocated into groups to receive dentures made using either traditional or simplified techniques. Individuals allocated to the traditional arm had a final impression taken in a custom-made tray, a face-bow recording and a semi-adjustable articulator was used, with articulator remount after delivery. Those in the simplified-technique group had impressions taken in stock trays, no face-bow recording and a mono-plane articulator was used, with no articulator remount after delivery.

Outcome measure The main outcome was the patients' ratings on $100 \mathrm{~mm}$ Visual analogue scale (VAS) of overall general satisfaction with their set of dentures. Secondary outcomes were their VAS ratings of the maxillary and mandibular dentures alone for satisfaction, comfort, stability, aesthetics, ability to speak, ease of cleaning, and ability to chew soft white bread, hard cheese, raw carrot, sausage, steak, raw apple and lettuce. The clinical assessment of denture quality at the 6month recall was carried out by four prosthodontists who were blind both to group allocation and to the purpose of the study.

Results There were no significant differences between the two groups in patient ratings for overall satisfaction at 3 or 6 months Conclusions These results support the use of simplified techniques, which are easier to master and which should reduce treatment costs. Educators should consider these findings when designing curricula for prosthodontic training.
Address for correspondence: Yasuhiko Kawai, Nihon University School of Dentistry at Matsudo, Department of Gnatho-Oral Prosthetic Rehabilitation, 2-870-1, Sakaechonishi, Matsudo, Chiba 271-8587, Japan. E-mail: ykawai@mascat.nihon-u.ac.jp

\section{Commentary}

There is a wealth of opinion about best practice in complete denture treatment but few comparative studies of the effectiveness of treatment techniques. This well-designed RCT compared the effectiveness of two different clinical techniques. As the authors state, the results of the study have considerable potential educational and economic implications.

One group of subjects $(n=58)$ received dentures produced by a traditional technique, using casts mounted with the aid of a facebow recording on a semi-adjustable articulator. The casts were made from functional impressions taken in special trays made on primary casts. This technique approximates what is taught in most dental schools and has been recommended best practice. ${ }^{1}$ A second group $(n=61)$ were provided with dentures produced by a simplified technique (only primary impressions taken and set up on a simple hinge articulator). From 1950 until at least 1990, most complete dentures provided by general dental practitioners in UK were produced using techniques similar to this "simplified" technique used in the study. ${ }^{2}$ The assumption (challenged by the evidence from this study) has been that dentures produced by what is here called the traditional technique were "better".

Satisfaction with complete dentures is a complex phenomenon but can be determined readily by subjective assessment. Satisfaction is compared in this study by mean VAS scores for each group but this avoids the question of how many people in each group could be classed as dissatisfied with their new dentures.

Objective evaluation of denture quality is more difficult. In this study the value of using the simple, validated (though infrequently used) rating scale to detect differences between the two techniques is limited: this is because many of the parameters scored in the rating scale (eg, posterior tooth position, tongue space, anterior tooth selection and arrangement) were independent of the techniques being assessed. The results of this study are nevertheless important and challenging. The study addresses issues that have been contentious in prosthetic dentistry for many years. It may not please the specialist prosthodontist but highlights the need for evidence that the choice of prosthetic technique does matter. ${ }^{2}$

\section{Practice point}

- Simple complete denture-making techniques can provide patient satisfaction for many edentulous patients. More studies are needed to determine the relative efficacy of complete denture techniques.

\section{Robert Jagger \\ Department of Oral and Dental Science, Bristol Dental School, Bristol, UK \\ 1. British Society for the Study of Prosthetic Dentistry. Guidelines in Prosthetic and Implant Dentistry, London: Quintessence; 1996. \\ 2. McCord JF, McNally LM, Smith PW, Gray NJA. Does the nature of the definitive impression material influence the outcome of (mandibular) complete dentures? Eur J Prosthodont Rest Dent 2005; 13:105-108.}

Evidence-Based Dentistry (2006) 7, 12.

doi:10.1038/sj.ebd.6400382 